\title{
Diffraction problems for quasilinear parabolic systems with boundary intersecting interfaces
}

\section{Qi-Jian Tan ${ }^{*}$ and Chao-Yi Pan}

\section{"Correspondence:}

tanqjxxx@yahoo.com.cn

Department of Mathematics,

Chengdu Normal University,

Chengdu, 611130, P.R. China

\begin{abstract}
In this paper, we discuss the $n$-dimensional diffraction problem for weakly coupled quasilinear parabolic system on a bounded domain $\Omega$, where the interfaces $\Gamma_{k}$ $(k=1, \ldots, K-1)$ are allowed to intersect with the outer boundary $\partial \Omega$ and the coefficients of the equations are allowed to be discontinuous on the interfaces. The aim is to show the existence of solutions by approximation method. The approximation problem is a diffraction problem with interfaces, which do not intersect with $\partial \Omega$.
\end{abstract}

MSC: 35R05; 35K57; 35K65

Keywords: diffraction problem; quasilinear parabolic system; interface; approximation method

\section{Introduction}

Let $\Omega$ be a bounded domain in $\mathbb{R}^{n}$ with boundary $\partial \Omega(n \geq 1)$, and let $\Omega$ be partitioned into a finite number of subdomains $\Omega_{k}(k=1, \ldots, K)$ separated by $\Gamma_{k}$, where $\Gamma_{k}, k=1, \ldots, K-1$, are interfaces, which do not intersect with each other. For any $T>0$, set

$$
Q_{T}:=\Omega \times(0, T], \quad S_{T}:=\partial \Omega \times[0, T], \quad \Gamma:=\bigcup_{k=1}^{K-1} \Gamma_{k}, \quad \Gamma_{T}:=\Gamma \times[0, T] .
$$

In this paper, we consider the diffraction problem for quasilinear parabolic reactiondiffusion system in the form

$$
\left\{\begin{array}{l}
u_{t}^{l}-\mathfrak{L}^{l}\left(u^{l}\right)=g^{l}(x, t, \mathbf{u}) \quad\left((x, t) \in Q_{T}\right), \\
{\left[u^{l}\right]_{\Gamma_{T}}=0, \quad\left[a_{i j}^{l}\left(x, t, u^{l}\right) u_{x_{j}}^{l} \nu_{i}(x)\right]_{\Gamma_{T}}=0,} \\
u^{l}=\psi^{l}(x, t) \quad\left((x, t) \in S_{T} \cup\{\Omega \times\{0\}\}\right), l=1, \ldots, N
\end{array}\right.
$$

where $x=\left(x_{1}, \ldots, x_{n}\right), \mathbf{u}=\left(u^{1}, \ldots, u^{N}\right), u_{t}^{l}:=\partial u^{l} / \partial t, u_{x_{i}}^{l}:=\partial u^{l} / \partial x_{i}, u_{x}^{l}:=\left(u_{x_{1}}^{l}, \ldots, u_{x_{n}}^{l}\right)$,

$$
\mathfrak{L}^{l}\left(u^{l}\right):=\frac{\mathrm{d}}{\mathrm{d} x_{i}}\left(a_{i j}^{l}\left(x, t, u^{l}\right) u_{x_{j}}^{l}\right)+b_{j}^{l}\left(x, t, u^{l}\right) u_{x_{j}}^{l}, \quad l=1, \ldots, N
$$

repeated indices $i$ or $j$ indicate summation from 1 to $n, \boldsymbol{v}(x):=\left(v_{1}(x), \ldots, v_{n}(x)\right)$ is the unit normal vector to $\Gamma$ (the positive direction of $\boldsymbol{v}(x)$ is fixed in advance), the symbol $[\cdot]_{\Gamma_{T}}$

\section{Springer}

(c) 2013 Tan and Pan; licensee Springer. This is an Open Access article distributed under the terms of the Creative Commons Attribution License (http://creativecommons.org/licenses/by/2.0), which permits unrestricted use, distribution, and reproduction in any medium, provided the original work is properly cited. 
denotes the jump of a quantity across $\Gamma_{T}$, and the coefficients $a_{i j}^{l}\left(x, t, u^{l}\right), b_{j}^{l}\left(x, t, u^{l}\right)$ and $g^{l}(x, t, \mathbf{u})$ are allowed to be discontinuous on $\Gamma_{T}$. In the following, we refer to the conditions on $\Gamma_{T}$ in (1.1) as diffraction conditions.

The diffraction problems often appear in different fields of physics, ecology, and technics. In some of them, the interfaces are allowed to intersect with the outer boundary $\partial \Omega$ (see [1-5]). The linear diffraction problems have been treated by many researchers (see [1-10]). For the quasilinear parabolic and elliptic diffraction problems, when all of the interfaces $\Gamma_{k}$ do not intersect with $\partial \Omega$, the existence and uniqueness of the solutions have been investigated in [11-14] by Leray-Schauder principle and the method of upper and lower solutions. In this paper, we investigate the existence of solutions of (1.1) when the interfaces are allowed to intersect with $\partial \Omega$. In this case, because of the existence of the intersection of $\Gamma$ and $\partial \Omega$, the methods in [11-14] can not be extended. We shall show the existence of solutions by approximation method. The approximation problem is a diffraction problem with interfaces which do not intersect with $\partial \Omega$.

The plan of the paper is as follows. In Sect. 2, we give the notations, hypotheses and an example, and state the existence theorem of the solutions. Section 3 is devoted to the proof of the existence theorem.

\section{The hypotheses, main result and example}

\subsection{The notations, hypotheses and main result}

First, let us introduce more notations and function spaces.

For any set $S, \bar{S}$ denotes its closure. The symbol $\Omega^{\prime} \subset \subset \Omega$ means that $\Omega^{\prime} \subset \Omega$ and $\operatorname{dist}\left(\Omega^{\prime}, \partial \Omega\right)>0$.

Let

$$
\left\{\Gamma_{1}, \ldots, \Gamma_{K-1}\right\}=\left\{\Gamma_{1}^{*}, \ldots, \Gamma_{K_{0}-1}^{*}\right\} \cup\left\{\Gamma_{1}^{* *}, \ldots, \Gamma_{K-K_{0}}^{* *}\right\}
$$

where $\Gamma_{k^{\prime}}^{*}, k^{\prime}=1, \ldots, K_{0}-1$, intersect with the outer boundary $\partial \Omega$, and $\Gamma_{k^{\prime \prime}}^{* *}, k^{\prime \prime}=1, \ldots, K-$ $K_{0}$ do not intersect with $\partial \Omega$. Assume that the domain $\Omega$ is partitioned into subdomains $\Omega_{k^{\prime}}^{*}, k^{\prime}=1, \ldots, K_{0}$, separated by interfaces $\Gamma_{k^{\prime}}^{*}$, and partitioned into $\Omega_{k^{\prime \prime}}^{* *}, k^{\prime \prime}=1, \ldots, K-$ $K_{0}+1$, separated by $\Gamma_{k^{\prime \prime}}^{* *}$. The interface of $\Omega_{k^{\prime}}^{*}$ and $\Omega_{k^{\prime}+1}^{*}$ is $\Gamma_{k^{\prime}}^{*}$. Then $\bar{\Omega}=\bigcup_{k^{\prime}=1}^{K_{0}} \bar{\Omega}_{k^{\prime}}^{*}=$ $\bigcup_{k^{\prime \prime}=1}^{K-K_{0}+1} \bar{\Omega}_{k^{\prime \prime}}^{* *}$ Set

$$
\begin{aligned}
& Q_{k, T}:=\Omega_{k} \times(0, T] \quad \text { for } k=1, \ldots, K, \\
& \Gamma^{*}:=\bigcup_{k^{\prime}=1}^{K_{0}-1} \Gamma_{k^{\prime}}^{*}, \quad \Gamma^{* *}:=\bigcup_{k^{\prime \prime}=1}^{K-K_{0}} \Gamma_{k^{\prime \prime}}^{* *}, \quad \Gamma_{T}^{*}:=\Gamma^{*} \times[0, T], \quad \Gamma_{T}^{* *}:=\Gamma^{* *} \times[0, T], \\
& Q_{k^{\prime}, T}^{*}:=\Omega_{k^{\prime}}^{*} \times(0, T] \quad \text { for } k^{\prime}=1, \ldots, K_{0}, \\
& Q_{k^{\prime \prime}, T}^{* *}:=\Omega_{k^{\prime \prime}}^{* *} \times(0, T] \quad \text { for } k^{\prime \prime}=1, \ldots, K-K_{0}+1 .
\end{aligned}
$$

We see that $\Gamma_{T}=\Gamma_{T}^{*} \cup \Gamma_{T}^{* *}$.

$C^{\alpha}\left(\bar{Q}_{T}\right)$ is the spaces of Hölder continuous in $\bar{Q}_{T}$ with exponent $\alpha \in(0,1) . W_{2}^{1}(\Omega)$ and $W_{2}^{1,1}\left(Q_{T}\right)$ are the Hilbert spaces with scalar products $(v, w)_{W_{2}^{1}(\Omega)}=\int_{\Omega}\left(\nu w+v_{x_{i}} w_{x_{i}}\right) \mathrm{d} x$ and $(v, w)_{W_{2}^{1,1}\left(Q_{T}\right)}=\iint_{Q_{T}}\left(v w+v_{t} w_{t}+v_{x_{i}} w_{x_{i}}\right) \mathrm{d} x \mathrm{~d} t$, respectively. Let

$$
\stackrel{\circ}{W}_{2}^{1}(\Omega):=\left\{v \in W_{2}^{1}(\Omega),\left.v\right|_{x \in \partial \Omega}=0\right\}, \quad \stackrel{\circ}{W}_{2}^{1,1}\left(Q_{T}\right):=\left\{v \in W_{2}^{1,1}\left(Q_{T}\right),\left.v\right|_{(x, t) \in S_{T}}=0\right\} .
$$


For the vector functions with $N$-components we denote the above function spaces by $\mathcal{C}^{\alpha}\left(\bar{Q}_{T}\right), \mathcal{W}_{2}^{1}(\Omega), \mathcal{W}_{2}^{1,1}\left(Q_{T}\right), \stackrel{\circ}{\mathcal{W}}_{2}^{1}(\Omega)$ and $\stackrel{\circ}{\mathcal{W}}_{2}^{1,1}\left(Q_{T}\right)$, respectively.

Moreover, we recall the following.

Definition 2.1 (see $[13,15])$ Write $\mathbf{u}$ in the split form

$$
\mathbf{u}=\left(u^{l},[\mathbf{u}]_{a^{l}},[\mathbf{u}]_{b^{l}}\right)
$$

The vector function $\mathbf{g}(\cdot, \mathbf{u}):=\left(g^{1}(\cdot, \mathbf{u}), \ldots, g^{N}(\cdot, \mathbf{u})\right)$ is said to be mixed quasimonotone in $\mathfrak{B} \subset \mathbb{R}^{N}$ with index vector $\left(a^{1}, \ldots, a^{N}\right)$ if for each $l=1, \ldots, N$, there exist nonnegative integers $a^{l}, b^{l}$, satisfying

$$
a^{l}+b^{l}=N-1
$$

such that $g^{l}\left(\cdot, u^{l},[\mathbf{u}]_{a^{l}},[\mathbf{u}]_{b^{l}}\right)$ is nondecreasing in $[\mathbf{u}]_{a^{l}}$, and is nonincreasing in $[\mathbf{u}]_{b^{l}}$ for all $\mathbf{u} \in \mathfrak{B}$.

The following hypotheses will be used in this paper:

(H) (i) $\partial \Omega$ and $\Gamma_{k}, k=1, \ldots, K-1$, are of $C^{2+\alpha_{0}}$ for some exponent $\alpha_{0} \in(0,1)$ and there exist $\theta_{0} \in(0,1)$ and $\rho_{0}>0$ such that for every open ball $K_{\rho}$ centered at $x_{0} \in \partial \Omega$ and radius $\rho \leq \rho_{0}$,

$$
\operatorname{mes}\left(K_{\rho} \cap \Omega\right) \leq\left(1-\theta_{0}\right) \operatorname{mes} K_{\rho} .
$$

Assume that for each $k^{\prime}=1, \ldots, K_{0}-1$,

$$
\Gamma_{k^{\prime}}^{*}: \varphi_{k^{\prime}}^{*}(x)=0 \quad(x \in \bar{\Omega}),
$$

and

$$
\bigcup_{\tau=1}^{k^{\prime}} \bar{\Omega}_{\tau}^{*}-\Gamma_{k^{\prime}}^{*}=\left\{x: \varphi_{k^{\prime}}^{*}(x)<0\right\} \cap \bar{\Omega} .
$$

(ii) Assume that

$$
\left\{\begin{array}{l}
a_{i j}^{l}\left(x, t, u^{l}\right)=a_{i j, k^{\prime}}^{l}\left(x, t, u^{l}\right), \quad b_{j}^{l}\left(x, t, u^{l}\right)=b_{j, k^{\prime}}^{l}\left(x, t, u^{l}\right), \\
g^{l}(x, t, \mathbf{u})=g_{k^{\prime}}^{l}(x, t, \mathbf{u}) \quad\left((x, t) \in Q_{k^{\prime}, T}^{*}, \mathbf{u} \in \mathbb{R}^{N}\right), k^{\prime}=1, \ldots, K_{0}
\end{array}\right.
$$

where $a_{i j, k^{\prime}}^{l}\left(x, t, u^{l}\right)$ and $b_{j, k^{\prime}}^{l}\left(x, t, u^{l}\right)$ are defined on $\bar{Q}_{T} \times \mathbb{R}, g_{k^{\prime}}^{l}(x, t, \mathbf{u})$ are defined on $\bar{Q}_{T} \times \mathbb{R}^{N}$, and all of them are allowed to be discontinuous on $\Gamma_{T}^{* *}$.

(iii) There exist constant vectors $\mathbf{M}=\left(M^{1}, \ldots, M^{N}\right)$ and $\mathbf{m}=\left(m^{1}, \ldots, m^{N}\right), \mathbf{m} \leq \mathbf{M}$, such that

$$
\begin{cases}g_{k^{\prime}}^{l}\left(x, t, M^{l},[\mathbf{M}]_{a^{l}},[\mathbf{m}]_{b^{l}}\right) \leq 0 & \left((x, t) \in Q_{T}\right), \\ g_{k^{\prime}}^{l}\left(x, t, m^{l},[\mathbf{m}]_{a^{l}},[\mathbf{M}]_{b^{l}}\right) \geq 0 \quad\left((x, t) \in Q_{T}\right), & \\ m^{l} \leq \psi^{l}(x, t) \leq M^{l} & \quad\left((x, t) \in S_{T} \cup\{\Omega \times\{0\}\}\right), k^{\prime}=1, \ldots, K_{0}, l=1, \ldots, N,\end{cases}
$$


where $a^{l}, b^{l}$ are all independent of $k^{\prime}$. Let

$$
\mathfrak{S}:=\left\{\mathbf{u} \in \mathcal{C}\left(\overline{\mathcal{Q}}_{T}\right): \mathbf{m} \leq \mathbf{u} \leq \mathbf{M}\right\}
$$

The vector functions $\mathbf{g}_{k^{\prime}}(\cdot, \mathbf{u})=\left(g_{k^{\prime}}^{1}(\cdot, \mathbf{u}), \ldots, g_{k^{\prime}}^{N}(\cdot, \mathbf{u})\right), k^{\prime}=1, \ldots, K_{0}$, are mixed quasimonotone in $\mathfrak{S}$ with the same index vector $\left(a^{1}, \ldots, a^{N}\right)$.

(iv) For each $k^{\prime}=1, \ldots, K_{0}, k^{\prime \prime}=1, \ldots, K-K_{0}+1, l=1, \ldots, N, a_{i j, k^{\prime}}^{l}\left(x, t, u^{l}\right)$, $b_{j, k^{\prime}}^{l}\left(x, t, u^{l}\right) \in C^{1+\alpha_{0}}\left(\bar{Q}_{k^{\prime \prime}, T}^{* *} \times \mathbb{R}\right)(i, j=1, \ldots, n), g_{k^{\prime}}^{l}(x, t, \mathbf{u}) \in C^{1+\alpha_{0}}\left(\bar{Q}_{k^{\prime \prime}, T}^{* *} \times \mathfrak{S}\right)$.

There exist a positive nonincreasing function $v(\theta)$ and a positive nondecreasing function $\mu(\theta)$ for $\theta \in[0,+\infty)$ such that

$$
\begin{aligned}
& v\left(\left|u^{l}\right|\right) \sum_{i^{\prime}=1}^{n} \xi_{i^{\prime}}^{2} \leq a_{i j, k^{\prime}}^{l}\left(x, t, u^{l}\right) \xi_{i} \xi_{j} \leq \mu\left(\left|u^{l}\right|\right) \sum_{i^{\prime}=1}^{n} \xi_{i^{\prime}}^{2} \\
& a_{i j, k^{\prime}}^{l}=a_{j i, k^{\prime}}^{l}, \quad\left|a_{i j, k^{\prime}}^{l}\left(x, t, u^{l}\right) ; b_{j, k^{\prime}}^{l}\left(x, t, u^{l}\right)\right| \leq \mu\left(\left|u^{l}\right|\right), \quad i, j=1, \ldots, N .
\end{aligned}
$$

For each $l=1, \ldots, N, \psi^{l}(x, t) \in C^{\alpha_{0}}(\bar{\Xi} \times[0, T]) \cap W_{2}^{1,1}(\Xi \times(0, T))$ for some domain $\Xi$ with $\Omega \subset \subset \Xi, \psi^{l}(x, 0) \in C^{2+\alpha_{0}}\left(\bar{\Omega}_{k}\right)(k=1, \ldots, K)$, and the following compatibility condition on $\Gamma^{* *}$ holds:

$$
\left[a_{i j}^{l}\left(x, 0, \psi^{l}(x, 0)\right) \psi_{x_{j}}^{l}(x, 0) v_{i}(x)\right]_{\Gamma^{* *}}=0 .
$$

Definition 2.2 A function $\mathbf{u}$ is said to be a solution of (1.1) if $\mathbf{u}$ possesses the following properties: (i) For some $\alpha \in(0,1), \mathbf{u} \in \mathcal{C}^{\alpha}\left(\bar{Q}_{T}\right) \cap \mathcal{C}^{2,1}\left(Q_{k, T}\right), k=1, \ldots, K$. For any given $\Omega^{\prime} \subset \subset \Omega$ and $t^{\prime} \in(0, T)$, there exists $\alpha^{\prime}, 0<\alpha^{\prime}<1$, such that $\mathbf{u}_{t} \in \mathcal{C}^{\alpha^{\prime}}\left(\bar{\Omega}^{\prime} \times\left[t^{\prime}, T\right]\right)$ and $\mathbf{u}_{x_{j}} \in \mathcal{L}^{2}\left(Q_{T}\right) \cap \mathcal{C}^{\alpha^{\prime}}\left(\left(\bar{\Omega}^{\prime} \cap \bar{\Omega}_{k}\right) \times\left[t^{\prime}, T\right]\right), k=1, \ldots, K, j=1, \ldots, n$; (ii) $\mathbf{u}$ satisfies the equations in (1.1) for $(x, t) \in Q_{k, T}, k=1, \ldots, K$, the diffraction conditions for $(x, t) \in \Gamma_{T} \cap Q_{T}$ and the parabolic boundary conditions for $(x, t) \in S_{T} \cup(\Omega \times\{0\})$.

The main result in this paper is the following existence theorem.

Theorem 2.1 Let Hypothesis $(\mathrm{H})$ hold. Then problem (1.1) has a solution $\mathbf{u}$ in $\mathfrak{S}$.

\subsection{An example}

We next give an example satisfying the conditions in Hypothesis $(\mathrm{H})$.

Example 2.1 In problem (1.1), let

$$
\begin{aligned}
& n=2, \quad \varphi=\left(x_{1}\right)^{2}+\left(x_{2}\right)^{2}-100, \quad \varphi_{1}=x_{1}+\left(x_{2}\right)^{2}+1, \\
& \varphi_{2}=x_{1}-\left(x_{2}\right)^{2}-1, \quad \varphi_{3}=\left(x_{1}-4\right)^{2}+\left(x_{2}\right)^{2}-1, \\
& \partial \Omega: \varphi=0, \quad \Gamma_{1}: \varphi_{1}=0 \quad\left(x_{1} \in I_{1}\right), \quad \Gamma_{2}: \varphi_{2}=0 \quad\left(x_{1} \in I_{2}\right), \quad \Gamma_{3}: \varphi_{3}=0,
\end{aligned}
$$

where $I_{1}=[-(9 \sqrt{5}-1) / 2,-1]$ and $I_{2}=[1,(9 \sqrt{5}-1) / 2]$, and let

$$
\begin{aligned}
& \Omega: \varphi<0, \quad \Omega_{1}: \varphi<0, \varphi_{1}<0, \quad \Omega_{2}: \varphi<0, \varphi_{1}>0, \varphi_{2}<0, \\
& \Omega_{3}: \varphi<0, \varphi_{2}>0, \varphi_{3}>0, \quad \Omega_{4}: \varphi_{3}<0 .
\end{aligned}
$$


Figure 1 The example of the domain and the interfaces for $n=2$.

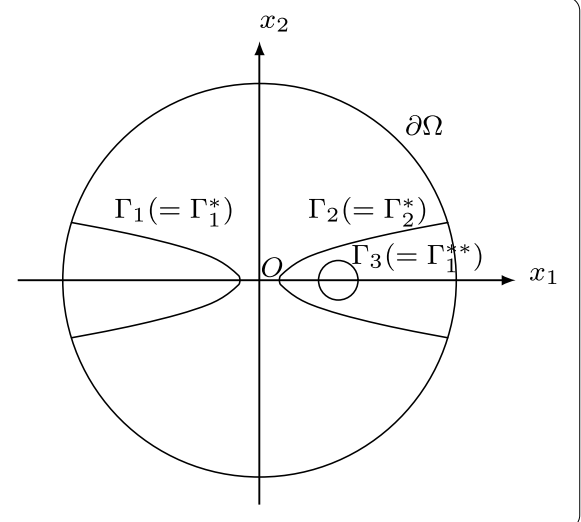

The outer boundary of domain is a circle of radius 10 with the center at the origin, whereas the interface curves are two parabolas and a smaller circle of radius 1 (see Figure 1). We see that $\Gamma_{1}$ and $\Gamma_{2}$ intersect with $\partial \Omega$, and $\Gamma_{3}$ does not.

For the coefficients of the equations and the boundary values in (1.1) we set

$$
\begin{aligned}
& a_{i j}^{l}\left(x, t, u^{l}\right)=\left\{\begin{array}{ll}
A_{k}^{l} E^{l}\left(u^{l}\right), & i=j, \\
0, & i \neq j
\end{array} \quad\left((x, t) \in Q_{k, T}, u^{l} \in \mathbb{R}\right), k=1,2,3,4, i, j=1,2,\right. \\
& b_{j}^{l}\left(x, t, u^{l}\right) \equiv 0 \quad\left((x, t) \in Q_{T}, u^{l} \in \mathbb{R}\right), j=1,2, \\
& g^{l}(x, t, \mathbf{u})=r_{k}^{l} u^{l} f_{k}^{l}(\mathbf{u}) \quad\left((x, t) \in Q_{k, T}, \mathbf{u} \in \mathbb{R}^{N}\right), k=1,2,3,4, \\
& \psi^{l}(x, t) \equiv o^{l}, \quad l=1, \ldots, N,
\end{aligned}
$$

where

$$
f_{k}^{l}(\mathbf{u})=1-\sum_{l^{\prime}=1}^{N} \delta_{l^{\prime}, k}^{l} u^{l^{\prime}} \quad \text { for } l=1, \ldots, N-1, \quad f_{k}^{N}(\mathbf{u})=1+\sum_{l^{\prime}=1}^{N-1} \delta_{l^{\prime}, k}^{N} u^{l^{\prime}}-\delta_{N, k}^{N} u^{N},
$$

$E^{l}\left(u^{l}\right) \in C^{2}(\mathbb{R})$ with $E^{l}\left(u^{l}\right) \geq v_{0}$, and $v_{0}, A_{k}^{l}, r_{k}^{l}, \delta_{l^{\prime}, k}^{l}$ and $o^{l}$ are all positive constants for $k=1,2,3,4, l, l^{\prime}=1, \ldots, N$.

Then

$$
\begin{aligned}
& \Gamma_{1}^{*}=\Gamma_{1}, \quad \Gamma_{2}^{*}=\Gamma_{2}, \quad \Omega_{1}^{*}=\Omega_{1}, \quad \Omega_{2}^{*}=\Omega_{2}, \quad \Omega_{3}^{*}: \varphi<0, \varphi_{2}>0, \\
& \Gamma_{1}^{* *}=\Gamma_{3}, \quad \Omega_{1}^{* *}: \varphi<0, \varphi_{3}>0, \quad \Omega_{2}^{* *}=\Omega_{4} .
\end{aligned}
$$

For each $l=1, \ldots, N$, let

$$
\begin{aligned}
& a_{i j, k^{\prime}}^{l}\left(x, t, u^{l}\right)=0 \quad\left((x, t) \in Q_{T}, u^{l} \in \mathbb{R}\right), i \neq j, i, j=1,2, k^{\prime}=1,2,3, \\
& a_{i i, k^{\prime}}^{l}\left(x, t, u^{l}\right)=A_{k^{\prime}}^{l} E^{l}\left(u^{l}\right) \quad\left((x, t) \in Q_{T}, u^{l} \in \mathbb{R}\right), i=1,2, k^{\prime}=1,2, \\
& a_{i i, 3}^{l}\left(x, t, u^{l}\right)=\left\{\begin{array}{ll}
A_{3}^{l} E^{l}\left(u^{l}\right) & \left((x, t) \in Q_{1, T}^{* *}, u^{l} \in \mathbb{R}\right), \\
A_{4}^{l} E^{l}\left(u^{l}\right) & \left((x, t) \in Q_{2, T}^{* *}, u^{l} \in \mathbb{R}\right),
\end{array} \quad i=1,2,\right. \\
& g_{k^{\prime}}^{l}(x, t, \mathbf{u})=r_{k^{\prime}}^{l} u_{k^{\prime}}^{l} f^{l}(\mathbf{u}) \quad\left((x, t) \in Q_{T}, \mathbf{u} \in \mathbb{R}^{N}\right), k^{\prime}=1,2,
\end{aligned}
$$




$$
g_{3}^{l}(x, t, \mathbf{u})= \begin{cases}r_{3}^{l} u^{l} f_{3}^{l}(\mathbf{u}) & \left((x, t) \in Q_{1, T}^{* *}, \mathbf{u} \in \mathbb{R}^{N}\right), \\ r_{4}^{l} u^{l} f_{4}^{l}(\mathbf{u}) & \left((x, t) \in Q_{2, T}^{* *}, \mathbf{u} \in \mathbb{R}^{N}\right) .\end{cases}
$$

We find that these functions satisfy (2.2) and the hypothesis (iv) of (H). Set $\mathbf{m}=(0, \ldots, 0)$. Then the requirements on $\mathbf{M}$ in (2.3) become

$$
\begin{aligned}
& 1-\delta_{l, k}^{l} M^{l} \leq 0, \quad M^{l} \geq o^{l}, l=1, \ldots, N-1 . \\
& 1+\sum_{l^{\prime}=1}^{N-1} \delta_{l^{\prime}, k}^{N} M^{l^{\prime}}-\delta_{N, k}^{N} M^{N} \leq 0, \quad M^{N} \geq o^{N} .
\end{aligned}
$$

It follows from these inequalities that there exist positive constant vector $\mathbf{M}$, such that $\mathbf{m}$ and $\mathbf{M}$ satisfy (2.3). Furthermore, the vector functions $\mathbf{g}_{k^{\prime}}(\cdot, \mathbf{u})=\left(g_{k^{\prime}}^{1}(\cdot, \mathbf{u}), \ldots, g_{k^{\prime}}^{N}(\cdot, \mathbf{u})\right)$, $k^{\prime}=1,2,3$, are mixed quasimonotone in $\mathfrak{S}$ with the same index vector $(0, \ldots, 0, N-1)$. The above arguments show that the conditions in Hypothesis $(\mathrm{H})$ can be satisfied.

\section{The proof of the existence theorem}

\subsection{Preliminaries}

Lemma 3.1 The following statements hold true:

(i) For any given $x \in \bar{\Omega}$, if $\varphi_{k_{0}^{\prime}}^{*}(x) \leq 0$ for some $k_{0}^{\prime} \in\left\{1, \ldots, K_{0}-2\right\}$, then

$$
\varphi_{\theta}^{*}(x)<0 \quad \text { for all } \theta \in\left\{k_{0}^{\prime}+1, \ldots, K_{0}-1\right\} \text {. }
$$

(ii) There exists a positive number $\varepsilon_{0}$ such that for any given $k^{\prime} \in\left\{2, \ldots, K_{0}-1\right\}$, if $1 \leq \theta \leq k^{\prime}-1$, then

$$
\varphi_{\theta}^{*}(x) \geq \varepsilon_{0} \quad \text { for all } x \in\left\{y: \varphi_{k^{\prime}}^{*}(y) \geq 0\right\} \cap \bar{\Omega} .
$$

Proof By (2.1), if $x \in \bar{\Omega}$ and $\varphi_{k_{0}^{\prime}}^{*}(x) \leq 0$, then $x \in \bigcup_{\tau=1}^{k_{0}^{\prime}} \bar{\Omega}_{\tau}^{*}$. Thus for each $\theta=k_{0}^{\prime}+1, \ldots, K_{0}-1$, $x \in \bigcup_{\tau=1}^{\theta} \bar{\Omega}_{\tau}^{*}-\Gamma_{\theta}^{*}$. Again by $(2.1)$ we get $\varphi_{\theta}^{*}(x)<0$. This proves the result in (i).

For any given $k^{\prime} \in\left\{2, \ldots, K_{0}-1\right\}$, if $x \in \bar{\Omega}$ and $\varphi_{k^{\prime}}^{*}(x) \geq 0$, then it follows from (i) that $\varphi_{\theta}^{*}(x)>0$ for all $\theta \in\left\{1, \ldots, k^{\prime}-1\right\}$. Since $\varphi_{\theta}^{*} \in C^{2+\alpha_{0}}$, there exist positive constants $\varepsilon_{k^{\prime}, \theta}$ such that

$$
\varphi_{\theta}^{*}(x) \geq \varepsilon_{k^{\prime}, \theta} \quad \text { for all } x \in\left\{y: \varphi_{k^{\prime}}^{*}(y) \geq 0\right\} \cap \bar{\Omega} .
$$

Hence, the conclusion in (ii) follows from the above relation by taking $\varepsilon_{0}:=\min _{k^{\prime}, \theta} \varepsilon_{k^{\prime}, \theta}$.

For an arbitrary $\varepsilon, 0<\varepsilon<\varepsilon_{0}$, let $s_{\varepsilon}=s_{\varepsilon}(\theta)$ be smooth function with values between 0 and 1 such that $\left|\frac{\mathrm{d}}{\mathrm{d} \theta} s_{\varepsilon}(\theta)\right| \leq C / \varepsilon$ for all $\theta \in \mathbb{R}, s_{\varepsilon}(\theta)=1$ for $\theta \leq 0$ and $s_{\varepsilon}(\theta)=0$ for $\theta \geq \varepsilon$. Define

$$
z_{\varepsilon, k^{\prime}}(x):=\left\{\begin{array}{l}
\prod_{\tau=1}^{K_{0}-1} s_{\varepsilon}\left(\varphi_{\tau}^{*}(x)\right) \quad(x \in \bar{\Omega}), k^{\prime}=1, \\
\prod_{\vartheta=1}^{K_{0}-1}\left[1-s_{\varepsilon}\left(\varphi_{\vartheta}^{*}(x)\right)\right] \quad(x \in \bar{\Omega}), k^{\prime}=K_{0}, \\
\prod_{\tau=k^{\prime}}^{K_{0}-1} s_{\varepsilon}\left(\varphi_{\tau}^{*}(x)\right) \prod_{\vartheta=1}^{k^{\prime}-1}\left[1-s_{\varepsilon}\left(\varphi_{\vartheta}^{*}(x)\right)\right] \quad(x \in \bar{\Omega}), k^{\prime}=2, \ldots, K_{0}-1 .
\end{array}\right.
$$


Lemma 3.2 $z_{\varepsilon, k^{\prime}}(x), k^{\prime}=1, \ldots, K_{0}$, are smooth functions with values between 0 and 1 , and possess the property

$$
\sum_{k^{\prime}=1}^{K_{0}} z_{\varepsilon, k^{\prime}}(x)=1 \quad(x \in \bar{\Omega}) .
$$

Let functions $\eta_{k^{\prime}}(x), k^{\prime}=1, \ldots, K_{0}$, be defined on $\bar{\Omega}$, and let

$$
\eta_{\varepsilon}(x)=\sum_{k^{\prime}=1}^{K_{0}} \eta_{k^{\prime}}(x) z_{\varepsilon, k^{\prime}}(x) \quad(x \in \bar{\Omega}) .
$$

Then for any $x \in \bar{\Omega}$,

$$
\eta_{\varepsilon}(x)=\left\{\begin{array}{l}
\eta_{1}(x) \quad \text { if } \varphi_{1}^{*}(x) \leq 0 \\
\eta_{K_{0}}(x) \quad \text { if } \varphi_{K_{0}-1}^{*}(x) \geq \varepsilon \\
\eta_{k^{\prime}}(x) \quad \text { if } \varphi_{k^{\prime}-1}^{*}(x) \geq \varepsilon \text { and } \varphi_{k^{\prime}}^{*}(x) \leq 0 \text { for some } k^{\prime} \in\left\{2, \ldots, K_{0}-1\right\} \\
\eta_{k^{\prime}-1}(x) s_{\varepsilon}\left(\varphi_{k^{\prime}-1}^{*}(x)\right)+\eta_{k^{\prime}}(x)\left[1-s_{\varepsilon}\left(\varphi_{k^{\prime}-1}^{*}(x)\right)\right] \\
\quad \text { if } 0<\varphi_{k^{\prime}-1}^{*}(x)<\varepsilon \text { for some } k^{\prime} \in\left\{2, \ldots, K_{0}-1\right\} .
\end{array}\right.
$$

Proof Since (3.2) is a special case of (3.4) with $\eta_{k^{\prime}}(x) \equiv 1$ for all $k^{\prime} \in\left\{1, \ldots, K_{0}\right\}$, we only prove (3.4).

Case 1. If $\varphi_{1}^{*}(x) \leq 0$, then the conclusion of (i) in Lemma 3.1 implies that $\varphi_{k^{\prime}}^{*}(x) \leq 0$ and $s_{\varepsilon}\left(\varphi_{k^{\prime}}^{*}(x)\right)=1$ for all $k^{\prime} \in\left\{1, \ldots, K_{0}-1\right\}$. (3.1) yields that $z_{\varepsilon, 1}(x)=1$ and $z_{\varepsilon, k^{\prime}}(x)=0$ for $k^{\prime} \geq 2$. These, together with (3.3), imply that $\eta_{\varepsilon}(x)=\eta_{1}(x)$.

Case 2. If $\varphi_{K_{0}-1}^{*}(x) \geq \varepsilon$, then the conclusion of (ii) in Lemma 3.1 shows that $\varphi_{k^{\prime}}^{*}(x) \geq \varepsilon$ and $s_{\varepsilon}\left(\varphi_{k^{\prime}}^{*}(x)\right)=0$ for all $k^{\prime} \in\left\{1, \ldots, K_{0}-1\right\}$. Hence, $z_{\varepsilon, K_{0}}(x)=1$ and $z_{\varepsilon, k^{\prime}}(x)=0$ for all $k^{\prime} \in$ $\left\{1, \ldots, K_{0}-1\right\}$. Again by (3.3) we get $\eta_{\varepsilon}(x)=\eta_{K_{0}}(x)$.

Case 3. If $\varphi_{k^{\prime}}^{*}(x) \leq 0$ and $\varphi_{k^{\prime}-1}^{*}(x) \geq \varepsilon$ for some $k^{\prime} \in\left\{2, \ldots, K_{0}-1\right\}$, then Lemma 3.1 yields that $\varphi_{\tau^{\prime}}^{*}(x) \leq 0, s_{\varepsilon}\left(\varphi_{\tau^{\prime}}^{*}(x)\right)=1$ for all $\tau^{\prime} \in\left\{k^{\prime}, \ldots, K_{0}-1\right\}$, and that $\varphi_{\tau^{\prime \prime}}^{*}(x) \geq \varepsilon, s_{\varepsilon}\left(\varphi_{\tau^{\prime \prime}}^{*}(x)\right)=0$ for all $\tau^{\prime \prime} \in\left\{1, \ldots, k^{\prime}-1\right\}$. Hence, $z_{\varepsilon, k^{\prime}}(x)=1$ and $z_{\varepsilon, \tau^{\prime}}(x)=0$ for $\tau^{\prime} \neq k^{\prime}$. Therefore, $\eta_{\varepsilon}(x)=$ $\eta_{k^{\prime}}(x)$.

Case 4. If $0<\varphi_{k^{\prime}-1}^{*}(x)<\varepsilon$ for some $k^{\prime} \in\left\{2, \ldots, K_{0}-1\right\}$, then it follows from Lemma 3.1 that $\varphi_{\tau^{\prime}}^{*}(x)>\varepsilon$ and $s_{\varepsilon}\left(\varphi_{\tau^{\prime}}^{*}(x)\right)=0$ for all $\tau^{\prime} \in\left\{1, \ldots, k^{\prime}-2\right\}$, and that $\varphi_{k^{\prime}}^{*}(x)<0$. Again by the conclusion of (i) in Lemma 3.1 we have $\varphi_{\tau^{\prime \prime}}^{*}(x)<0$ and $s_{\varepsilon}\left(\varphi_{\tau^{\prime \prime}}^{*}(x)\right)=1$ for all $\tau^{\prime \prime} \in\left\{k^{\prime}, \ldots, K_{0}-\right.$ $1\}$. Hence, $z_{\varepsilon, k^{\prime}}(x)=1-s_{\varepsilon}\left(\varphi_{k^{\prime}-1}^{*}(x)\right), z_{\varepsilon, k^{\prime}-1}(x)=s_{\varepsilon}\left(\varphi_{k^{\prime}-1}^{*}(x)\right)$ and $z_{\varepsilon, \tau}(x)=0$ for $\tau \neq k^{\prime}, k^{\prime}-1$. Thus, $\eta_{\varepsilon}(x)=\eta_{k^{\prime}-1}(x) s_{\varepsilon}\left(\varphi_{k^{\prime}-1}^{*}(x)\right)+\eta_{k^{\prime}}(x)\left[1-s_{\varepsilon}\left(\varphi_{k^{\prime}-1}^{*}(x)\right)\right]$.

\subsection{The approximation problem of (1.1)}

In this subsection, we construct a problem to approximate (1.1).

For each $l=1, \ldots, N$, let

$$
\left\{\begin{array}{l}
a_{i j \varepsilon}^{l}=a_{i j \varepsilon}^{l}\left(x, t, u^{l}\right):=\sum_{k^{\prime}=1}^{K_{0}} a_{i j, k^{\prime}}^{l}\left(x, t, u^{l}\right) z_{\varepsilon, k^{\prime}}(x), \\
b_{j \varepsilon}^{l}=b_{j \varepsilon}^{l}\left(x, t, u^{l}\right):=\sum_{k^{\prime}=1}^{K_{0}} b_{j, k^{\prime}}^{l}\left(x, t, u^{l}\right) z_{\varepsilon, k^{\prime}}(x), \\
g_{\varepsilon}^{l}=g_{\varepsilon}^{l}(x, t, \mathbf{u}):=\sum_{k^{\prime}=1}^{K_{0}} g_{k^{\prime}}^{l}(x, t, \mathbf{u}) z_{\varepsilon, k^{\prime}}(x) \quad\left((x, t) \in Q_{T}\right) .
\end{array}\right.
$$


It follows from hypothesis (iv) of (H), (3.2) and (3.5) that $a_{i j \varepsilon}^{l}\left(x, t, u^{l}\right), b_{j \varepsilon}^{l}\left(x, t, u^{l}\right)$ are in $C^{1+\alpha_{0}}\left(\bar{Q}_{k^{\prime \prime}, T}^{* *} \times \mathbb{R}\right)(i, j=1, \ldots, n), g_{\varepsilon}^{l}(x, t, \mathbf{u})$ is in $C^{1+\alpha_{0}}\left(\bar{Q}_{k^{\prime \prime}, T}^{* *} \times \mathfrak{S}\right)\left(k^{\prime \prime}=1, \ldots, K-K_{0}+1\right)$, the vector function $\mathbf{g}_{\varepsilon}(\cdot, \mathbf{u})=\left(g_{\varepsilon}^{1}(\cdot, \mathbf{u}), \ldots, g_{\varepsilon}^{N}(\cdot, \mathbf{u})\right)$ is mixed quasimonotone in $\mathfrak{S}$ with index vector $\left(a^{1}, \ldots, a^{N}\right)$, and

$$
\begin{aligned}
& v\left(\left|u^{l}\right|\right) \sum_{i^{\prime}=1}^{n} \xi_{i^{\prime}}^{2} \leq a_{i j \varepsilon}^{l}\left(x, t, u^{l}\right) \xi_{i} \xi_{j} \leq \mu\left(\left|u^{l}\right|\right) \sum_{i^{\prime}=1}^{n} \xi_{i^{\prime}}^{2}, \\
& a_{i j \varepsilon}^{l}=a_{j i \varepsilon}^{l}, \quad\left|a_{i j \varepsilon}^{l}\left(x, t, u^{l}\right) ; b_{j \varepsilon}^{l}\left(x, t, u^{l}\right)\right| \leq \mu\left(\left|u^{l}\right|\right), \quad i, j=1, \ldots, n .
\end{aligned}
$$

We note that the functions $a_{i j \varepsilon}^{l}\left(x, t, u^{l}\right), b_{j \varepsilon}^{l}\left(x, t, u^{l}\right)$ and $g_{\varepsilon}^{l}(x, t, \mathbf{u})$ are continuous on $\Gamma_{T}^{*}$, and are allowed to be discontinuous on $\Gamma_{T}^{* *}$.

For each $k^{\prime \prime}=1, \ldots, K-K_{0}$, there exists $\Omega_{\tau_{k^{\prime \prime}}}^{*}$ such that $\Gamma_{k^{\prime \prime}}^{* *} \subset \Omega_{\tau_{k^{\prime \prime}}}^{*}$. Take two subdomains $B_{k^{\prime \prime}, 1}, B_{k^{\prime \prime}, 2}$ satisfying $\Gamma_{k^{\prime \prime}}^{* *} \subset B_{k^{\prime \prime}, 1} \subset \subset B_{k^{\prime \prime}, 2} \subset \subset \Omega_{\tau_{k^{\prime \prime}}}^{*}$. Let $\lambda_{k^{\prime \prime}}=\lambda_{k^{\prime \prime}}(x)$ be an arbitrary smooth function taking values in $[0,1]$ such that $\lambda_{k^{\prime \prime}}=0$ for $x \notin \Omega_{\tau_{k^{\prime \prime}}}^{*}$ and $\lambda_{k^{\prime \prime}}=1$ for $x \in B_{k^{\prime \prime}, 2}$. Set

$$
\begin{aligned}
\psi_{\varepsilon}^{l} & =\psi_{\varepsilon}^{l}(x, t) \\
& :=\int_{|x-y| \leq \varepsilon} \omega(|x-y|)\left(1-\sum_{k^{\prime \prime}=1}^{K-K_{0}} \lambda_{k^{\prime \prime}}(y)\right) \psi^{l}(y, t) \mathrm{d} y+\sum_{k^{\prime \prime}=1}^{K-K_{0}} \lambda_{k^{\prime \prime}}(x) \psi^{l}(x, t)
\end{aligned}
$$

with a sufficiently smooth nonnegative averaging kernel $\omega(|\xi|)$ that is equal to zero for $|\xi| \geq 1$ and is such that $\int_{\mid \xi \leq 1} \omega(\xi) \mathrm{d} \xi=1$. Then from the hypothesis (iv) of (H) and [1, Chapter II] we know that for each $l=1, \ldots, N, \psi_{\varepsilon}^{l}(x, t)$ is in $C^{\alpha_{0}}\left(\bar{Q}_{T}\right) \cap W_{2}^{1,1}\left(Q_{T}\right), \psi_{\varepsilon}^{l}(x, 0)$ is in $C^{2+\alpha_{0}}\left(\bar{\Omega}_{k^{\prime \prime}}^{* *}\right)\left(k^{\prime \prime}=1, \ldots, K-K_{0}+1\right), \psi_{\varepsilon}^{l} \rightarrow \psi^{l}$ in $C^{\alpha_{0}}\left(\bar{Q}_{T}\right)$ and $\psi_{\varepsilon}^{l} \rightarrow \psi^{l}$ in $W_{2}^{1,1}\left(Q_{T}\right)$. Thus,

$$
\left\|\psi_{\varepsilon}^{l}(x, t)\right\|_{C^{\alpha}\left(\bar{Q}_{T}\right)}+\left\|\psi_{\varepsilon}^{l}\right\|_{W_{2}^{1,1}\left(Q_{T}\right)} \leq \mu_{1},
$$

where $\mu_{1}$ is a positive constant, independent of $\varepsilon$. Furthermore, (3.4), (3.5) and (3.8) show that for small enough $\varepsilon$,

$$
\begin{aligned}
& a_{i j \varepsilon}^{l}\left(x, t, u^{l}\right)=a_{i j, \tau_{k^{\prime \prime}}}^{l}\left(x, t, u^{l}\right), \\
& \psi_{\varepsilon}^{l}(x, t)=\psi^{l}(x, t) \quad\left((x, t) \in B_{k^{\prime \prime}, 1} \times[0, T]\right), k^{\prime \prime}=1, \ldots, K-K_{0} .
\end{aligned}
$$

These, together with (2.6), imply that

$$
\left[a_{i j \varepsilon}\left(x, 0, \psi_{\varepsilon}^{l}(x, 0)\right) \psi_{\varepsilon x_{j}}^{l}(x, 0) v_{i}\right]_{\Gamma^{* *}}=0
$$

For any given $\varepsilon, 0<\varepsilon<\varepsilon_{0}$, consider the approximation diffraction problem of (1.1)

$$
\left\{\begin{array}{l}
u_{t}^{l}-\mathfrak{L}_{\varepsilon}^{l}\left(u^{l}\right)=g_{\varepsilon}^{l}(x, t, \mathbf{u}) \quad\left((x, t) \in Q_{T}\right), \\
{\left[u^{l}\right]_{\Gamma_{T}^{* *}}=0, \quad\left[a_{i j \varepsilon}^{l}\left(x, t, u^{l}\right) u_{x_{j}}^{l} v_{i}(x)\right]_{\Gamma_{T}^{* *}}=0,} \\
u^{l}=\psi_{\varepsilon}^{l}(x, t) \quad\left((x, t) \in S_{T} \cup\{\Omega \times\{0\}\}\right), l=1, \ldots, N,
\end{array}\right.
$$


where

$$
\mathfrak{L}_{\varepsilon}^{l}\left(u^{l}\right):=\frac{\mathrm{d}}{\mathrm{d} x_{i}}\left(a_{i j \varepsilon}^{l}\left(x, t, u^{l}\right) u_{x_{j}}^{l}\right)+b_{j \varepsilon}^{l}\left(x, t, u^{l}\right) u_{x_{j}}^{l} .
$$

We note that the interfaces in $(3.11)$ are $\Gamma_{k^{\prime \prime}}^{* *}\left(k^{\prime \prime}=1, \ldots, K-K_{0}\right)$ which do not intersect with $\partial \Omega$. In view of (3.10), the compatibility condition on $\Gamma^{* *}$ holds.

Proposition 3.1 Problem (3.11) has a unique piecewise classical solution $\mathbf{u}_{\varepsilon}=\mathbf{u}_{\varepsilon}(x, t)$ in $\mathfrak{S}$ possessing the following properties:

$$
\begin{array}{lcl}
\mathbf{u}_{\varepsilon} \in \mathcal{C}^{\alpha}\left(\bar{Q}_{T}\right), & \mathbf{u}_{\varepsilon t} \in \mathcal{C}^{\alpha, \alpha / 2}\left(\bar{Q}_{T}\right), & \mathbf{u}_{\varepsilon x_{j}} \in \mathcal{C}^{\alpha, \alpha / 2}\left(\bar{Q}_{k^{\prime \prime}, T}^{* *}\right) \quad(\alpha \in(0,1)), \\
\mathbf{u}_{\varepsilon x_{j} t} \in \mathcal{L}^{2}\left(Q_{T}\right), & \mathbf{u}_{\varepsilon x_{i} x_{j}} \in \mathcal{C}\left(Q_{k^{\prime \prime}, T}^{* *}\right), & k^{\prime \prime}=1, \ldots, K-K_{0}+1 .
\end{array}
$$

Proof Problem (3.11) is a special case of [13, problem (1.1)] without time delays. Formulas (2.3) and (3.5) show that $\tilde{\mathbf{u}}=\mathbf{M}, \hat{\mathbf{u}}=\mathbf{m}$ are a pair of bounded and coupled weak upper and lower solutions of (3.11) in the sense of [13, Definition 2.2]. We find that the conditions of [13, Theorem 4.1] are all fulfilled. Then from [13, Theorem 4.1], we obtain that problem (3.11) has a unique piecewise classical solution $\mathbf{u}_{\varepsilon}=\mathbf{u}_{\varepsilon}(x, t)$ in $\mathfrak{S}$ possessing the properties in (3.12).

\subsection{The uniform estimates of $\mathbf{u}_{\varepsilon}$}

In the following discussion, let $K_{\rho}$ be an arbitrary open ball of radius $\rho$ with center at $x^{0}$, and let $Q_{\rho}$ be an arbitrary cylinder of the form $K_{\rho} \times\left[t^{0}-\rho^{2}, t^{0}\right]$.

For each $l=1, \ldots, N$, consider the equality $\int_{t_{0}}^{t} \int_{\Omega}\left[u_{\varepsilon t}^{l}-\mathfrak{L}_{\varepsilon}^{l}\left(u_{\varepsilon}^{l}\right)\right] \eta^{l} \mathrm{~d} x \mathrm{~d} t=\int_{t_{0}}^{t} \int_{\Omega} g_{\varepsilon}^{l}(x, t$, $\left.\mathbf{u}_{\varepsilon}\right) \eta^{l} \mathrm{~d} x \mathrm{~d} t$ for any function $\eta^{l}=\eta^{l}(x, t)$ from $\stackrel{\circ}{W}_{2}^{1,1}\left(Q_{T}\right)$ with $\operatorname{ess} \sup _{Q_{T}}\left|\eta^{l}\right|<\infty$ and for any $t_{0}, t$ from [0,T]. In view of $\mathbf{u}_{\varepsilon} \in \mathfrak{S}$, it follows from (3.6), (3.7), (3.9) and the formula of integration by parts that

$$
\begin{aligned}
& \left.\int_{\Omega} u_{\varepsilon}^{l} \eta^{l} \mathrm{~d} x\right|_{t_{0}} ^{t}+\int_{t_{0}}^{t} \int_{\Omega}\left[-u_{\varepsilon}^{l} \eta_{t}^{l}+a_{i j \varepsilon}^{l}\left(x, t, u_{\varepsilon}^{l}\right) u_{\varepsilon x_{j}}^{l} \eta_{x_{i}}^{l}\right] \mathrm{d} x \mathrm{~d} t \\
& =\int_{t_{0}}^{t} \int_{\Omega}\left[-b_{j \varepsilon}^{l}\left(x, t, u_{\varepsilon}^{l}\right) u_{\varepsilon x_{j}}^{l}+g_{\varepsilon}^{l}\left(x, t, \mathbf{u}_{\varepsilon}\right)\right] \eta^{l} \mathrm{~d} x \mathrm{~d} t \\
& \leq C \int_{t_{0}}^{t} \int_{\Omega}\left[\left|u_{\varepsilon x}^{l}\right|+1\right]\left|\eta^{l}\right| \mathrm{d} x \mathrm{~d} t .
\end{aligned}
$$

Similarly, for any $\phi^{l} \in \stackrel{\circ}{W}_{2}^{1}(\Omega)$ and for every $t \in[0, T]$ we get

$$
\int_{\Omega} a_{i j \varepsilon}^{l}\left(x, t, u_{\varepsilon}^{l}\right) u_{\varepsilon x_{j}}^{l} \phi_{x_{i}}^{l} \mathrm{~d} x=\int_{\Omega}\left[-u_{\varepsilon t}^{l}-b_{j \varepsilon}^{l}\left(x, t, u_{\varepsilon}^{l}\right) u_{\varepsilon x_{j}}^{l}+g_{\varepsilon}^{l}\left(x, t, \mathbf{u}_{\varepsilon}\right)\right] \phi^{l} \mathrm{~d} x \mathrm{~d} t .
$$

Lemma 3.3 There exist constants $\alpha_{1}\left(0<\alpha_{1}<1\right)$ and $C$ depending only on $M_{0}$ (:= $\max (|\mathbf{M}|,|\mathbf{m}|)), \rho_{0}, \theta_{0}, \alpha_{0}, v\left(M_{0}\right), \mu\left(M_{0}\right)$ and $\mu_{1}$, independent of $\varepsilon$, such that

$$
\begin{aligned}
& \left\|u_{\varepsilon}^{l}\right\|_{C^{\alpha_{1}, \alpha_{1} / 2}\left(\bar{Q}_{T}\right)} \leq C, \\
& \left\|u_{\varepsilon x}^{l}\right\|_{L^{2}\left(Q_{T}\right)} \leq C, \quad l=1, \ldots, N .
\end{aligned}
$$


Proof (3.16) follows from (3.14), (3.6), (3.7), (3.9) and [1, Chapter V, Theorem 1.1 and Remark 1.2]. Setting $\eta^{l}=u_{\varepsilon}^{l}-\psi_{\varepsilon}^{l}$ in (3.14) and using Cauchy's inequality, we can obtain (3.17).

Lemma 3.4 For any given $k_{1}^{\prime} \in\left\{1, \ldots, K_{0}\right\}$, let $D_{1} \subset \subset \Omega_{k_{1}^{\prime}}^{*}$ and $t^{\prime} \in(0, T)$. Then there exist positive constants $\alpha_{2}\left(0<\alpha_{2}<1\right)$ and $C\left(d_{1}^{\prime}, t^{\prime}\right)$ depending only on $d_{1}^{\prime}\left(:=\operatorname{dist}\left(D_{1}, \partial \Omega_{k_{1}^{\prime}}^{*}\right)\right), t^{\prime}$ and the parameters $M_{0}, \rho_{0}, \theta_{0}, \alpha_{0}, v\left(M_{0}\right), \mu\left(M_{0}\right)$ and $\mu_{1}$, independent of $\varepsilon$, such that for any $\Omega_{k^{\prime \prime}}^{* *}$ satisfying $D_{1} \cap \Omega_{k^{\prime \prime}}^{* *} \neq \emptyset$,

$$
\begin{aligned}
& \left\|u_{\varepsilon x_{j}}^{l}\right\|_{C^{\alpha_{2}}\left(\left(\overline{D_{1} \cap \Omega_{k^{\prime \prime}}^{* *}}\right) \times\left[t^{\prime}, T\right]\right)} \leq C\left(d_{1}^{\prime}, t^{\prime}\right), \quad j=1, \ldots, n, l=1, \ldots, N, \\
& \left\|u_{\varepsilon t}^{l}\right\|_{C^{\alpha_{2}\left(\bar{D}_{1} \times\left[t^{\prime}, T\right]\right)}} \leq C\left(d_{1}^{\prime}, t^{\prime}\right), \quad l=1, \ldots, N .
\end{aligned}
$$

For any given $k \in\{1, \ldots, K\}$, let $\Omega^{\prime \prime} \subset \subset \Omega_{k}$ and $t^{\prime \prime} \in(0, T)$. Then there exist positive constants $\alpha_{3}\left(0<\alpha_{3}<1\right)$ and $C\left(d^{\prime \prime}, t^{\prime \prime}\right)$ depending only on $d^{\prime \prime}\left(:=\operatorname{dist}\left(\Omega^{\prime \prime}, \partial \Omega_{k}\right)\right)$, $t^{\prime \prime}$ and the parameters $M_{0}, \rho_{0}, \theta_{0}, \alpha_{0}, \nu\left(\left(M_{0}\right)\right), \mu\left(M_{0}\right)$ and $\mu_{1}$, such that

$$
\left\|u_{\varepsilon}^{l}\right\|_{C^{2+\alpha_{3}, 1+\alpha_{3} / 2\left(\bar{\Omega}^{\prime \prime} \times\left[t^{\prime \prime}, T\right]\right)}} \leq C\left(d^{\prime \prime}, t^{\prime \prime}\right), \quad l=1, \ldots, N
$$

Proof Choose a subdomain $B$ satisfying $D_{1} \subset \subset B \subset \subset \Omega_{k_{1}^{\prime}}^{*}$. (3.4) and (3.5) show that for small enough $\varepsilon$,

$$
\left\{\begin{array}{l}
a_{i j \varepsilon}^{l}\left(x, t, u^{l}\right)=a_{i j, k_{1}^{\prime}}^{l}\left(x, t, u^{l}\right), \\
b_{j \varepsilon}^{l}\left(x, t, u^{l}\right)=b_{j, k_{1}^{\prime}}^{l}\left(x, t, u^{l}\right), \\
g_{\varepsilon}^{l}(x, t, \mathbf{u})=g_{k_{1}^{\prime}}^{l}(x, t, \mathbf{u}) \quad((x, t) \in B \times(0, T]), l=1, \ldots, N .
\end{array}\right.
$$

Then the same proofs as those of [13, formulas (3.30) and (3.31)] give (3.18) and (3.19). If $\Omega^{\prime \prime} \subset \subset \Omega_{k}$, then $\Omega^{\prime \prime} \subset \subset \Omega_{k_{1}^{\prime}}^{*} \cap \Omega_{k^{\prime \prime}}^{* *}$ for some $k_{1}^{\prime} \in\left\{1, \ldots, K_{0}\right\}, k^{\prime \prime} \in\left\{1, \ldots, K-K_{0}+1\right\}$. Hence, the conclusion in (3.20) follows from (3.18), (3.19), (3.21) and the same argument as that for [13, formula (3.37)].

In the rest of this subsection, let $k_{2}^{\prime}$ be an arbitrary fixed number in $\left\{1, \ldots, K_{0}-1\right\}$, and let $D_{2} \subset \subset \Omega$ be an arbitrary fixed subdomain satisfying $D_{2} \cap \Gamma_{k_{2}^{\prime}}^{*} \neq \emptyset, \bar{D}_{2} \cap\left(\Gamma_{k_{2}^{\prime}-1}^{*} \cup \Gamma_{k_{2}^{\prime}+1}^{*}\right)=$ $\emptyset$ and $\bar{D}_{2} \cap \Gamma^{* *}=\emptyset$. We next investigate the uniform estimates in the neighborhood of $\Gamma_{k_{2}^{\prime}}^{*} \cap \bar{D}_{2}$. Let $x^{0}$ be any point of $\Gamma_{K_{2}^{\prime}}^{*} \cap \bar{D}_{2}$. [2, Chapter 3, Section 16] and [13] show that there exists a ball $K_{\rho}$ with center at $x^{0}$ such that we can straighten $\Gamma_{K_{2}^{\prime}}^{*} \cap K_{\rho}$ out by introducing a local coordinate system $y=y(x)$. Our assumptions concerning $\Gamma$ imply that we can divide $\Gamma_{k_{2}^{\prime}}^{*} \cap \bar{D}_{2}$ into a finite number of pieces and to introduce for each of them coordinates $y$. Since the investigations in the rest of this subsection are local properties, we can assume without loss of generality that the interface $\Gamma_{k_{2}^{\prime}}^{*}$ lies in the plane $x_{n}=0$. Then by (3.4), when $(x, t) \in D_{2} \times[0, T]$ the coefficients of problem (3.11) can be represented in the form

$$
\left\{\begin{array}{l}
a_{i j \varepsilon}^{l}\left(x, t, u^{l}\right)=a_{i j, k_{2}^{\prime}}^{l}\left(x, t, u^{l}\right) s_{\varepsilon}\left(x_{n}\right)+a_{i j, k_{2}^{\prime}+1}^{l}\left(x, t, u^{l}\right)\left[1-s_{\varepsilon}\left(x_{n}\right)\right], \\
b_{j \varepsilon}^{l}\left(x, t, u^{l}\right)=b_{j, k_{2}^{\prime}}^{l}\left(x, t, u^{l}\right) s_{\varepsilon}\left(x_{n}\right)+b_{j, k_{2}^{\prime}+1}^{l}\left(x, t, u^{l}\right)\left[1-s_{\varepsilon}\left(x_{n}\right)\right], \\
g_{\varepsilon}^{l}(x, t, \mathbf{u})=g_{k_{2}^{\prime}}^{l}(x, t, \mathbf{u}) s_{\varepsilon}\left(x_{n}\right)+g_{k_{2}^{\prime}+1}^{l}(x, t, \mathbf{u})\left[1-s_{\varepsilon}\left(x_{n}\right)\right], \quad l=1, \ldots, N,
\end{array}\right.
$$


and the diffraction conditions on $\Gamma_{T}^{*}$ in problem (1.1) can be represented in the form

$$
\left[u^{l}\right]_{\Gamma_{T}^{*}}=0, \quad\left[a_{n j}^{l}\left(x, t, u^{l}\right) u_{x_{j}}^{l}\right]_{\Gamma_{T}^{*}}=0, \quad l=1, \ldots, N .
$$

Lemma 3.5 Let $t^{\prime} \in(0, T)$. Then there exist positive constants $\alpha_{4}\left(0<\alpha_{4}<1\right)$ and $C\left(d_{2}^{\prime}, t^{\prime}\right)$ depending only on $d_{2}^{\prime}\left(:=\min \left\{\operatorname{dist}\left(D_{2}, \partial \Omega\right), \operatorname{dist}\left(D_{2}, \Gamma_{k_{2}^{\prime}-1}^{*} \cup \Gamma_{k_{2}^{\prime}+1}^{*}\right), \operatorname{dist}\left(D_{2}, \Gamma^{* *}\right)\right\}\right), t^{\prime}$, and the parameters $M_{0}, \rho_{0}, \theta_{0}, \alpha_{0}, v\left(M_{0}\right), \mu\left(M_{0}\right)$ and $\mu_{1}$, independent of $\varepsilon$, such that

$$
\begin{aligned}
& \left\|u_{\varepsilon x_{s}}^{l}\right\|_{C^{\alpha_{4}}\left(\bar{D}_{2} \times\left[t^{\prime}, T\right]\right)} \leq C\left(d_{2}^{\prime}, t^{\prime}\right), \quad s=1, \ldots, n-1, \\
& \left\|\varpi_{\varepsilon, n}^{l}\right\|_{C^{\alpha_{4}}\left(\bar{D}_{2} \times\left[t^{\prime}, T\right]\right)} \leq C\left(d_{2}^{\prime}, t^{\prime}\right), \quad \varpi_{\varepsilon, n}^{l}:=a_{n j \varepsilon}^{l}\left(x, t, u_{\varepsilon}^{l}\right) u_{\varepsilon x_{j}}^{l}, \\
& \left\|u_{\varepsilon t}^{l}\right\|_{C^{\alpha_{4}}\left(\bar{D}_{2} \times\left[t^{\prime}, T\right]\right)} \leq C\left(d_{2}^{\prime}, t^{\prime}\right), \quad l=1, \ldots, N .
\end{aligned}
$$

Proof It follows from (3.22) and Hypothesis (H) that

$$
\begin{aligned}
& \left|\frac{\partial a_{i j \varepsilon}^{l}\left(x, t, u^{l}\right)}{\partial x_{s}} ; \frac{\partial a_{i j \varepsilon}^{l}}{\partial t} ; \frac{\partial a_{i j \varepsilon}^{l}}{\partial u^{l}}\right|+\left|\frac{\partial b_{j \varepsilon}^{l}\left(x, t, u^{l}\right)}{\partial x_{s}} ; \frac{\partial b_{j \varepsilon}^{l}}{\partial t} ; \frac{\partial b_{j \varepsilon}^{l}}{\partial u^{l}}\right|+\left|\frac{\partial g_{\varepsilon}^{l}(x, t, \mathbf{u})}{\partial x_{s}} ; \frac{\partial g_{\varepsilon}^{l}}{\partial t} ; \frac{\partial g_{\varepsilon}^{l}}{\partial u^{l^{\prime}}}\right| \\
& \quad \leq C \quad\left((x, t) \in \bar{D}_{2} \times[0, T], \mathbf{u} \in \mathfrak{S}\right), s=1, \ldots, n-1, l, l^{\prime}=1, \ldots, N
\end{aligned}
$$

and from the equations in (3.11) that

$$
\begin{gathered}
\left|\frac{\mathrm{d}}{\mathrm{d} x_{n}}\left(a_{n j \varepsilon}^{l}\left(x, t, u_{\varepsilon}^{l}\right) u_{\varepsilon x_{j}}^{l}\right)\right| \leq C\left(\left|u_{\varepsilon t}^{l}\right|+\sum_{s=1}^{n-1} \sum_{j=1}^{n}\left|u_{\varepsilon x_{j} x_{s}}^{l}\right|+\left|u_{\varepsilon x}^{l}\right|^{2}+1\right) \\
\left((x, t) \in \bar{D}_{2} \times[0, T]\right), l=1, \ldots, N .
\end{gathered}
$$

Then using (3.13), (3.15), (3.22), (3.27) and (3.28), we can prove (3.24)-(3.26) by a slight modification of the proofs of [13, formulas (3.30) and (3.31)]. The detailed proofs are omitted.

\subsection{The proof of Theorem 2.1}

From estimates (3.16), (3.17) and the Arzela-Ascoli theorem it follows that we can find a subsequence (we retain the same notation for it) $\left\{\mathbf{u}_{\varepsilon}\right\}$ such that $\left\{\mathbf{u}_{\varepsilon}\right\}$ converges in $\mathcal{C}\left(\bar{Q}_{T}\right)$ to $\mathbf{u}$ and $\left\{\mathbf{u}_{\varepsilon x_{j}}\right\}$ converges weakly in $\mathcal{L}^{2}\left(Q_{T}\right)$ to $\mathbf{u}_{x_{j}}$ for each $j=1, \ldots, n$. Then $\mathbf{u} \in \mathcal{C}^{\alpha_{1}}\left(\bar{Q}_{T}\right)$ and $\mathbf{u}_{x_{j}} \in \mathcal{L}^{2}\left(Q_{T}\right)$. Furthermore, the parabolic boundary conditions for $\mathbf{u}_{\varepsilon}$ in (3.11) imply that $\mathbf{u}$ satisfies the parabolic boundary conditions in (1.1).

For any given $k \in\{1, \ldots, K\}$, and for any $\Omega^{\prime \prime} \subset \subset \Omega_{k}, t^{\prime \prime} \in(0, T)$, (3.20) yields that there exists a subsequence $\left\{\mathbf{u}_{\varepsilon^{\prime}}\right\}$ (denoted by $\left\{\mathbf{u}_{\varepsilon}\right\}$ still) such that $\left\{\mathbf{u}_{\varepsilon}\right\}$ converges in $\mathcal{C}^{2,1}\left(\bar{\Omega}^{\prime \prime} \times\right.$ $\left.\left[t^{\prime \prime}, T\right]\right)$ to $\mathbf{u}$. By letting $\varepsilon \rightarrow 0$, from (3.21) and the equations $u_{\varepsilon t}^{l}-\mathfrak{L}_{\varepsilon}^{l}\left(u_{\varepsilon}^{l}\right)=g_{\varepsilon}^{l}\left(x, t, \mathbf{u}_{\varepsilon}\right)$ in (3.11) we get that

$$
u_{t}^{l}-\mathfrak{L}^{l}\left(u^{l}\right)=g^{l}(x, t, \mathbf{u}) \quad\left((x, t) \in \Omega^{\prime \prime} \times\left[t^{\prime \prime}, T\right]\right), l=1, \ldots, N
$$

Since $\Omega^{\prime \prime}$ and $t^{\prime \prime}$ are arbitrary, then $\mathbf{u}$ satisfies the equations in (3.11) for $(x, t) \in Q_{k, T}$.

For any given $k_{1}^{\prime} \in\left\{1, \ldots, K_{0}\right\}$ and for any $D_{1} \subset \subset \Omega_{k_{1}^{\prime}}^{*}, t^{\prime} \in(0, T)$, we see from (3.18), (3.19) that there exists a subsequence $\left\{\mathbf{u}_{\varepsilon^{\prime}}\right\}$ (denoted by $\left\{\mathbf{u}_{\varepsilon}\right\}$ still) such that for each $j=1, \ldots, n$ 
and for any $\Omega_{k^{\prime \prime}}^{* *}$ satisfying $D_{1} \cap \Omega_{k^{\prime \prime}}^{* *} \neq \emptyset,\left\{\mathbf{u}_{\varepsilon x_{j}}\right\}$ converges in $\left.\mathcal{C}\left(\overline{D_{1} \cap \Omega_{k^{\prime \prime}}^{* *}}\right) \times\left[t^{\prime}, T\right]\right)$ to $\mathbf{u}_{x_{j}}$, and $\left\{\mathbf{u}_{\varepsilon t}\right\}$ converges in $\mathcal{C}\left(\bar{D}_{1} \times\left[t^{\prime}, T\right]\right)$ to $\mathbf{u}_{t}$. Hence

$$
\mathbf{u}_{x_{j}} \in \mathcal{C}^{\alpha_{2}}\left(\left(\overline{D_{1} \cap \Omega_{k^{\prime \prime}}^{* *}}\right) \times\left[t^{\prime}, T\right]\right), \quad \mathbf{u}_{t} \in \mathcal{C}^{\alpha_{2}}\left(\bar{D}_{1} \times\left[t^{\prime}, T\right]\right) .
$$

By letting $\varepsilon \rightarrow 0$ we conclude from (3.21) and the diffraction conditions on $\Gamma_{T}^{* *}$ for $\mathbf{u}_{\varepsilon}$ in (3.11) that

$$
\left[u^{l}\right]_{\Gamma_{T}^{* *} \cap Q_{T}}=0, \quad\left[a_{i j}^{l}\left(x, t, u^{l}\right) u_{x_{j}}^{l} v_{i}(x)\right]_{\Gamma_{T}^{* *} \cap Q_{T}}=0, \quad l=1, \ldots, N .
$$

For any given $k_{2}^{\prime} \in\left\{1, \ldots, K_{0}-1\right\}$ and $D_{2} \subset \subset \Omega$ satisfying $D_{2} \cap \Gamma_{k_{2}^{\prime}}^{*} \neq \emptyset, \bar{D}_{2} \cap\left(\Gamma_{k_{2}^{\prime}-1}^{*} \cup\right.$ $\left.\Gamma_{k_{2}^{\prime}+1}^{*}\right)=\emptyset$ and $\bar{D}_{2} \cap \Gamma^{* *}=\emptyset$, the estimates (3.24)-(3.26) imply that for any given $t^{\prime} \in(0, T)$ there exists a subsequence $\left\{\mathbf{u}_{\varepsilon^{\prime}}\right\}$ (denoted by $\left\{\mathbf{u}_{\varepsilon}\right\}$ still) such that for each $s=1, \ldots, n-1$, $l=1, \ldots, N$,

$$
\begin{aligned}
& u_{\varepsilon x_{s}}^{l} \rightarrow u_{x_{s}}^{l}, \quad u_{\varepsilon t}^{l} \rightarrow u_{t}^{l}, \\
& \varpi_{\varepsilon, n}^{l}=a_{n j \varepsilon}^{l}\left(x, t, u_{\varepsilon}^{l}\right) u_{\varepsilon x_{j}}^{l} \rightarrow \varpi^{l} \quad \text { in } C\left(\bar{D}_{2} \times\left[t^{\prime}, T\right]\right) .
\end{aligned}
$$

Then

$$
u_{x_{s}}^{l}, u_{t}^{l}, \varpi^{l} \in C^{\alpha_{4}}\left(\bar{D}_{2} \times\left[t^{\prime}, T\right]\right) .
$$

We next show that $\varpi^{l}=a_{n j}^{l}\left(x, t, u^{l}\right) u_{x_{j}}^{l}$. For any $\eta=\eta(x, t) \in L^{2}\left(D_{2} \times\left(t^{\prime}, T\right)\right)$,

$$
\begin{aligned}
\int_{t^{\prime}}^{T} & \int_{D_{2}}\left[a_{n j \varepsilon}^{l}\left(x, t, u_{\varepsilon}^{l}\right) u_{\varepsilon x_{j}}^{l}-a_{n j}^{l}\left(x, t, u^{l}\right) u_{x_{j}}^{l}\right] \eta \mathrm{d} x \mathrm{~d} t \\
= & \int_{t^{\prime}}^{T} \int_{D_{2}}\left(a_{n j \varepsilon}^{l}\left(x, t, u_{\varepsilon}^{l}\right)-a_{n j \varepsilon}^{l}\left(x, t, u^{l}\right)\right) u_{\varepsilon x_{j}}^{l} \eta \mathrm{d} x \mathrm{~d} t \\
& +\int_{t^{\prime}}^{T} \int_{D_{2}}\left(a_{n j \varepsilon}^{l}\left(x, t, u^{l}\right)-a_{n j}^{l}\left(x, t, u^{l}\right)\right) u_{\varepsilon x_{j}}^{l} \eta \mathrm{d} x \mathrm{~d} t \\
& +\int_{t^{\prime}}^{T} \int_{D_{2}} a_{n j}^{l}\left(x, t, u^{l}\right)\left(u_{\varepsilon x_{j}}^{l}-u_{x_{j}}^{l}\right) \eta \mathrm{d} x \mathrm{~d} t \\
:= & I_{\varepsilon, 1}^{l}+I_{\varepsilon, 2}^{l}+I_{\varepsilon, 3}^{l} .
\end{aligned}
$$

By (3.27), (3.17), we get

$$
\begin{aligned}
\left|I_{\varepsilon, 1}^{l}\right| & \leq C\left\|\left(u_{\varepsilon}^{l}-u^{l}\right) \eta\right\|_{L^{2}\left(D_{2} \times\left(t^{\prime}, T\right)\right)}\left\|u_{\varepsilon x_{j}}^{l}\right\|_{L^{2}\left(D_{2} \times\left(t^{\prime}, T\right)\right)} \\
& \leq C^{\prime}\left\|\left(u_{\varepsilon}^{l}-u^{l}\right) \eta\right\|_{L^{2}\left(D_{2} \times\left(t^{\prime}, T\right)\right)} \rightarrow 0 \quad \text { as } \varepsilon \rightarrow 0,
\end{aligned}
$$

and by (2.2), (3.22),

$$
\begin{aligned}
\left|I_{\varepsilon, 2}^{l}\right| & =\left|\int_{t^{\prime}}^{T} \int_{D_{2} \cap\left\{x \mid 0 \leq x_{n} \leq \varepsilon\right\}}\left(a_{n j, k_{2}^{\prime}}^{l}\left(x, t, u^{l}\right)-a_{n j, k_{2}^{\prime}+1}^{l}\left(x, t, u^{l}\right)\right) s_{\varepsilon}\left(x_{n}\right) u_{\varepsilon x_{j}}^{l} \eta \mathrm{d} x \mathrm{~d} t\right| \\
& \leq C\left\|u_{\varepsilon x_{j}}^{l}\right\|_{L^{2}\left(D_{2} \times(0, T)\right)}\left\{\int_{t^{\prime}}^{T} \int_{D_{2} \cap\left\{x \mid 0 \leq x_{n} \leq \varepsilon\right\}} \eta^{2} \mathrm{~d} x \mathrm{~d} t\right\}^{1 / 2}
\end{aligned}
$$




$$
\begin{aligned}
& \leq C^{\prime}\left\{\int_{t^{\prime}}^{T} \int_{D_{2} \cap\left\{x \mid 0 \leq x_{n} \leq \varepsilon\right\}} \eta^{2} \mathrm{~d} x \mathrm{~d} t\right\}^{1 / 2} \\
& \rightarrow 0 \text { as } \varepsilon \rightarrow 0 .
\end{aligned}
$$

Since $\left\{\mathbf{u}_{\varepsilon x_{j}}\right\}$ converges weakly in $\mathcal{L}^{2}\left(Q_{T}\right)$ to $\mathbf{u}_{x_{j}}$ for each $j=1, \ldots, n$, then $I_{\varepsilon, 3}^{l} \rightarrow 0$ as $\varepsilon \rightarrow 0$. Hence, $\varpi_{\varepsilon, n}^{l}=a_{n j \varepsilon}^{l}\left(x, t, u_{\varepsilon}^{l}\right) u_{\varepsilon x_{j}}^{l}$ converges weakly in $L^{2}\left(D_{2} \times\left(t^{\prime}, T\right)\right)$ to $a_{n j}^{l}\left(x, t, u^{l}\right) u_{x_{j}}^{l}$ for each $j=1, \ldots, n$. This, together with (3.31), implies that

$$
\varpi^{l}=a_{n j}^{l}\left(x, t, u^{l}\right) u_{x_{j}}^{l} \in C^{\alpha_{4}}\left(\bar{D}_{2} \times\left[t^{\prime}, T\right]\right)
$$

and $\mathbf{u}$ satisfies the diffraction conditions on $\Gamma_{T}^{*} \cap Q_{T}$ in (3.23).

In view of (3.30) $\mathbf{u}$ satisfies the diffraction conditions on $\Gamma_{T} \cap Q_{T}$ in (1.1). Furthermore, (3.29), (3.32) and (3.33) imply that for any $k \in\{1, \ldots, K\}, \Omega^{\prime} \subset \subset \Omega$,

$$
\mathbf{u}_{x_{j}} \in \mathcal{C}^{\alpha}\left(\left(\overline{\Omega^{\prime} \cap \Omega_{k}}\right) \times\left[t^{\prime}, T\right]\right), \quad \mathbf{u}_{t} \in \mathcal{C}^{\alpha}\left(\overline{\Omega^{\prime}} \times\left[t^{\prime}, T\right]\right), \quad j=1, \ldots, n
$$

for some $\alpha \in(0,1)$. Therefore, $\mathbf{u}$ is a solution of (1.1). This completes the proof of Theorem 2.1.

\section{Competing interests}

The authors declare that they have no competing interests.

\section{Authors' contributions}

All authors read and approved the final manuscript.

\section{Acknowledgements}

Dedicated to Professor Hari M Srivastava.

The authors would like to thank the reviewers and the editors for their valuable suggestions and comments. The work was supported by the research fund of Department of Education of Sichuan Province (10ZC127) and the research fund of Chengdu Normal University (CSYXM12-06).

\section{Received: 19 January 2013 Accepted: 9 April 2013 Published: 22 April 2013}

\section{References}

1. Ladyzenskaya, OA, Solonnikov, VA, Ural'ceva, NN: Linear and Quasilinear Equations of Parabolic Type. Am. Math. Soc., Providence (1968)

2. Ladyzenskaya, OA, Ural'ceva, NN: Linear and Quasilinear Elliptic Equations. Academic Press, New York (1968)

3. Ladyzenskaya, OA, Ryvkind, VJ, Ural'ceva, NN: Solvability of diffraction problems in the classical sense. Tr. Mat. Inst. Steklova 92, 116-146 (1966) (in Russian)

4. Yi, FH: Global classical solution of Muskat free boundary problem. J. Math. Anal. Appl. 288, 442-461 (2003)

5. Druet, PE: Global Lipschitz continuity for elliptic transmission problems with a boundary intersecting interface. Weierstrass Institute for Applied Analysis and Stochastics, WIAS Preprint No. 1571 (2010)

6. Kamynin, LI: On the linear Verigin problem. Dokl. Akad. Nauk SSSR 150, 1210-1213 (1963) (in Russian)

7. Kamynin, Ll: The method of heat potentials for a parabolic equation with discontinuous coefficients. Sib. Mat. Zh. 4, 1071-1105 (1963) (in Russian)

8. Chen, Z, Zou, Z: Finite element methods and their convergence for elliptic and parabolic interface problems. Numer. Math. 79, 175-202 (1998)

9. Huang, J, Zou, J: Some new a priori estimates for second-order elliptic and parabolic interface problems. J. Differ. Equ. $184,570-586(2002)$

10. Evans, LC: A free boundary problem: the flow of two immiscible fluids in a one-dimmensional porous medium. I. Indiana Univ. Math. J. 26, 914-932 (1977)

11. Rivkind, V, Ural'tseva, N: Classical solvability and linear schemas for the approximated solutions of the diffraction problem for quasilinear elliptic and parabolic equations. Mat. Zametki 3, 69-111 (1972) (in Russian)

12. Boyadjiev, G, Kutev, N: Diffraction problems for quasilinear reaction-diffusion systems. Nonlinear Anal. 55, 905-926 (2003)

13. Tan, QJ: Systems of quasilinear parabolic equations with discontinuous coefficients and continuous delays. Adv. Differ. Equ. 2011, Article ID 925173 (2011). doi:10.1155/2011/925173

14. Tan, QJ, Leng, ZJ: The method of upper and lower solutions for diffraction problems of quasilinear elliptic reaction-diffusion systems. J. Math. Anal. Appl. 380, 363-376 (2011)

15. Pao, CV: Nonlinear Parabolic and Elliptic Equations. Plenum Press, New York (1992) 
doi:10.1186/1687-2770-2013-99

Cite this article as: Tan and Pan: Diffraction problems for quasilinear parabolic systems with boundary intersecting interfaces. Boundary Value Problems 2013 2013:99.

Submit your manuscript to a SpringerOpen ${ }^{\circ}$ journal and benefit from:

- Convenient online submission

- Rigorous peer review

- Immediate publication on acceptance

- Open access: articles freely available online

- High visibility within the field

- Retaining the copyright to your article

Submit your next manuscript at $\gg$ springeropen.com 